\title{
La estructura geológica de la península Ibérica y sus aguas termales
}

Carlos Martín Escorza *

\section{INTRODUCCIÓN}

La Tierra tiene una estructuración interna que de forma simple podemos suponer como con un gran núcleo caliente en su interior con temperaturas que pueden superar $\operatorname{los} 4.000{ }^{\circ} \mathrm{C}$, y una superficie a temperaturas medias entre $15-20^{\circ} \mathrm{C}$. Estos hechos indican claramente que se está produciendo un flujo de energia calorífica desde dentro hacia el exterior. Este flujo centrífugo hubiera enfriado ya el planeta si nó fuera por la existencia del aporte de un calor extra proveniente de la desintegración de los materiales radioactivos que se encuentran en la corteza terrestre. Estas fuentes que implementan calor al sistema se encuentran sobre todo en las rocas graníticas de la corteza continental. Todo el conjunto de estos procesos producen en el planeta un flujo superficial general medio de unos $50 \mathrm{mWm}^{-2}$, aunque en determinadas áreas aumenta este valor hasta superarlo en más de veinte veces.

Las áreas en que el valor del flujo es mayor corresponde a las zonas en las que existen superpuestos o por separado algunos procesos tales como una elevada sismicidad, actividad volcánica reciente o en tiempos geológicos recientes y/o fenómenos de deformación orogénica. Estas zonas son en las que podemos esperar encontrar más probablemente surgencias de aguas minero-medicinales y/o termales (A.M.M.T.). Para comprender dicha relación es necesario recordar que las aguas que denominamos termales provienen de aguas meteóricas que se han infiltrado

* Museo Nacional de Ciencias Naturales C.S.I.C. 
entre las rocas. Si este flujo hídrico subterráneo llega a profundizar, a través de planos de fracturas con las adecuadas condiciones como conductos, o bien sin tener que alcanzar valores mínimos de profundidad el área tiene un elevado flujo geotérmico, se produce un calentamiento de esas aguas. Aprovechando el mismo u otro plano de fractura esas aguas ya calientes ascienden de nuevo a la superficie dando lugar a las A.M.M.T.

La relación entre la estructura geológica de la península lbérica y la distribución general de las surgencias de A.M.M.T. es un tema tratado con cierta periodicidad (SÁnchez LozANo 1914; HeRnÁndez-PACHECo 1949; LóPEZ dE AzCONA 1963; Menduiña 1978). Por nuestra parte con esta nueva aportación se pretende poner de manifiesto con más detalle esta relación, ya que contamos para ello: con más información sobre los puntos con estas características, aparecida en recientes años; con nuevos datos sobre la geología de las áreas con ellos relacionados; y con los medios informáticos necesarios que hacen posible un tratamiento de esta gran cantidad de datos para de forma seleccionada darles una expresión gráfica en la que basar las conclusiones.

También debemos decir que esta base de datos, aunque amplia, todavía es incompleta, ya que de forma continua encontramos más referencias a fenómenos de este tipo.

\section{BREVE DESCRIPCIÓN DE LA GEOLOGÍA PENINSULAR}

En un ejercicio ciertamente complicado de sintesis baste decir aquí que la península lbérica es un mosaico de piezas geológicas sedimentadas en muy diferentes épocas, desde los tiempos Paleozoicos (que se inician hace 540 M.A.), o aún quizás Proterozoicos, hasta los últimos depósitos fluviales cuaternarios. Durante esta gran amplitud de tiempo han tenido lugar diferentes etapas de deformación tectónica las más destacadas de las cuales tuvieron sus efectos tranformadores hace unos 350 M.A. (Orogenia Hercínica), que afectó también a una buena parte del actual continente europeo, y otra muy posterior en la que su etapa principal aconteció hace unos 30 M.A. (Orogenia Alpina), afectando asimismo a buena parte de la Europa meridional y norte de África. La orogenia Alpina tiene su continuación hoy en los movimientos y tensiones que se desarrollan en buena parte de esta región. 
La más antigua de estas dos orogenias dio lugar a transformaciones y deformaciones, ocurridas en diferentes niveles de la corteza. Las rocas entonces existentes fueron plegadas, fracturadas, algunas transformadas en granitos y todas ellas de diversa manera alteradas sus posiciones y composiciones. Estas rocas se muestran ahora visibles por erosión en el Pirineo central y oriental, en las áreas centrales de la Meseta (Sistema Central) y occidentales (Galicia, Extremadura, Andalucía occidental) de la península y tienen desde entonces impresas en sus masas las grandes fracturas que se formaron como consecuencia de las tensiones que los fenómenos tectónicos les impusieron.

Durante la Orogenia Alpina tuvieron lugar acontecimientos tectónicos de importancia desigual. Los más enérgicos y violentos sucedieron en las zonas pirenaica y bética, mientras que en el interior de la Meseta la existencia de los citados macizos cristalinos, ya rígidos, exhumados del interior desde que fueran formados durante la orogenia Hercínica, no permitieron que la deformación fuera tan intensa en los materiales sedimentados sobre ellos.

Se ha observado en muchas áreas que durante la Orogenia Alpina se pusieron en juego también las fracturas ya existentes desde el Hercínico, ya que ellas fueron y son estructuras más favorables, por preexistentes, a la amortiguación y transmisión de las tensiones que se han sucedido o pueden ir desarrollándose en el futuro.

Los efectos de esta última Orogenia todavía están teniendo lugar y en algunas áreas se manifiestan en actividad sísmica y de movimientos de masas rocosas. Tenemos constancia además que durante algunos periodos del Cuaternario en algunas zonas (Campos de Calatrava, Olot) han ocurrido procesos volcánicos de cierta importancia.

Esta actividad tectónica tiene una distribución inhomogénea en la península Ibérica. La región Bética, Galicia y el Pirineo son las zonas donde desde hace miles de años se viene desarrollando con más impetu y se manifiesta por la existencia en esas regiones de movimientos sísmicos de consideración.

La introducción, desde hace sólo unos veinte años, del Modelo de Tectónica de Placas para explicar muchos de estos fenómenos nos hacen ver hoy a esta porción pétrea como una «masa poligonal de cuatro lados" que tiene que adaptarse a los acontecimientos de los respectivos cuatro grandes conjuntos geológicos que la enmarcan: al complejo Mediterráneo; a la expansión del Atlántico; a la adyacente Placa Europea, a la que estamos literalmente pegados por el Pirineo, y a la gran Placa Africana, cuya dinámica ha marcado los caracteres geológicos de la región Bética.

Por otra parte resulta de interés observar cómo se distribuyen los pun- 
tos de afloramiento de esas aguas anómalas ya sea por su temperatura a través de la cual pueden ser aprovechadas para explotaciones geotérmicas (ALBERT Y BANDA 1984), como por su aparente relación de coincidencia con que en ocasiones se presentan con determinados yacimientos minerales en las mismas regiones (CRUZ-SANJULIÁN y GaRCiA-ROSSELL 1975; Menduiña 1978).

\section{Un mapa base de referencia}

Para mostrar la relación entre la distribución de los puntos en los que hay noticias de fuentes o manantiales minero-medicinales y la estructura geológica de la península lbérica se ha diseñado un mapa geológico base. Este mapa es el resultado de un compromiso entre la numerosa información geológica que se conoce y que sería necesario en buena parte incorporar y la elección de los mínimos datos posibles que se requieran para la finalidad que perseguimos aquí. Se ha indicado la distribución de los dos grandes conjuntos de rocas en que puede, en sintesis extrema, dividirse la peninsula lbérica:

- Por una parte, las rocas granítico-metamórficas, cristalinas o pizarrosas, que ocupan una buena parte de su mitad occidental, las zonas axiales de las cordilleras Ibérica, Pirineos y Bética. Una gran proporción de estas rocas fueron generadas o transformadas durante los procesos que tuvieron lugar durante la Orogenia Hercínica. Para la Cordillera Bética hay áreas que tienen su origen durante la Orogenia Alpina.

- Las sedimentarias que desde el Mesozoico hasta la actualidad se han depositado, tanto en ambientes marinos como continentales, en diferentes cuencas y luego en su mayor parte se encuentran deformadas por la Orogenia Alpina. Constituidas en una gran proporción por arenas, calizas, arcillas y yesos, ocupan grandes áreas del centro, mitad oriental y sur de la península.

En el mapa-base también se ha recogido la situación de varias fallas. Estos accidentes tectónicos son numerosos a escalas tanto centimétrica como kilométrica, pasando por las intermedias; en este esquema básico se han recogido las que podemos considerar como importantes, haciendo rápida salvedad de que a esta o cualquier otra selección siempre sería posible añadir otro elemento de este tipo. Por claridad y según la experiencia del autor las fracturas y fallas representadas aquí deben ser in- 
cluidas en cualquier caso, y en su mayor parte se hallan asimisrno recogidas por JULIVERT et al. (1974).

Puesto que estamos refiriéndonos a procesos actuales parece adecuado, sino necesario, señalar en el mapa-base la localización de los principales núcleos de actividad sísmica, pues estos muestran a veces estrecha relación con la temperatura de las surgencias de aguas y su aparición, y/o desaparición, durante algunos terremotos. Por ello se han recogido las isosistas de diferentes valores de intensidad, a partir de $\mathrm{Vl}$, hasta el valor máximo, $\mathrm{X}$, conocido en la península (MezcuA y MARTínez SOLARES 1983).

Quizás deberiamos haber indicado también en el mapa-base las dos áreas volcánicas de actividad ignea durante el Cuaternario: los Campos de Calatrava, en Ciudad Real, y el campo de Olot, en Girona. Pero por su carácter relativamente puntual será sólo suficiente por ahora con esta citación y después su posterior advertencia cuando se necesite.

Para los objetivos perseguidos aquí todos estos elementos señalados en la figura 1, pueden ser los suficientes para encuadrar y relacionar la distribución de las fuentes minero-medicinales en la estructura geológica de la península lbérica.

\section{EXTRACCIÓN DE LOS DATOS}

Recogiendo datos de los catálogos existentes y actualizando parte de dichos datos, así como teniendo en cuenta los de Portugal y Francia meridional, se ha elaborado, con motivo de esta reunión, un fichero de datos de las aguas termales $\mathrm{y} / \mathrm{o}$ mineralizadas que nos permite no sólo un inventario actualizado para la península, sino su aprovechamiento informático para la elaboración de mapas temáticos. Ajustando esta información a la estructura geológica básica de la península pretendemos mostrar la relación entre ambos fenómenos desglosando lo más posible los afloramientos de aguas según sus diversas características (temperatura y algunos caracteres químicos fundamentalmente).

Para la elaboración de esta Base de Datos se han consultado dos Guías Oficiales (1927, 1948) de balnearios de España; Enciclopedia Universal Ilustrada «Espasa» (1930); todas las Memorias de los Mapas Geológicos a escala 1:50.000, realizados por el Instituto Geológico y Minero de España (hoy Instituto Tecnológico y Geominero de España, I.T.G.E.) publicadas hasta el año 1960 (Primera Serie), en las cuales era frecuente 


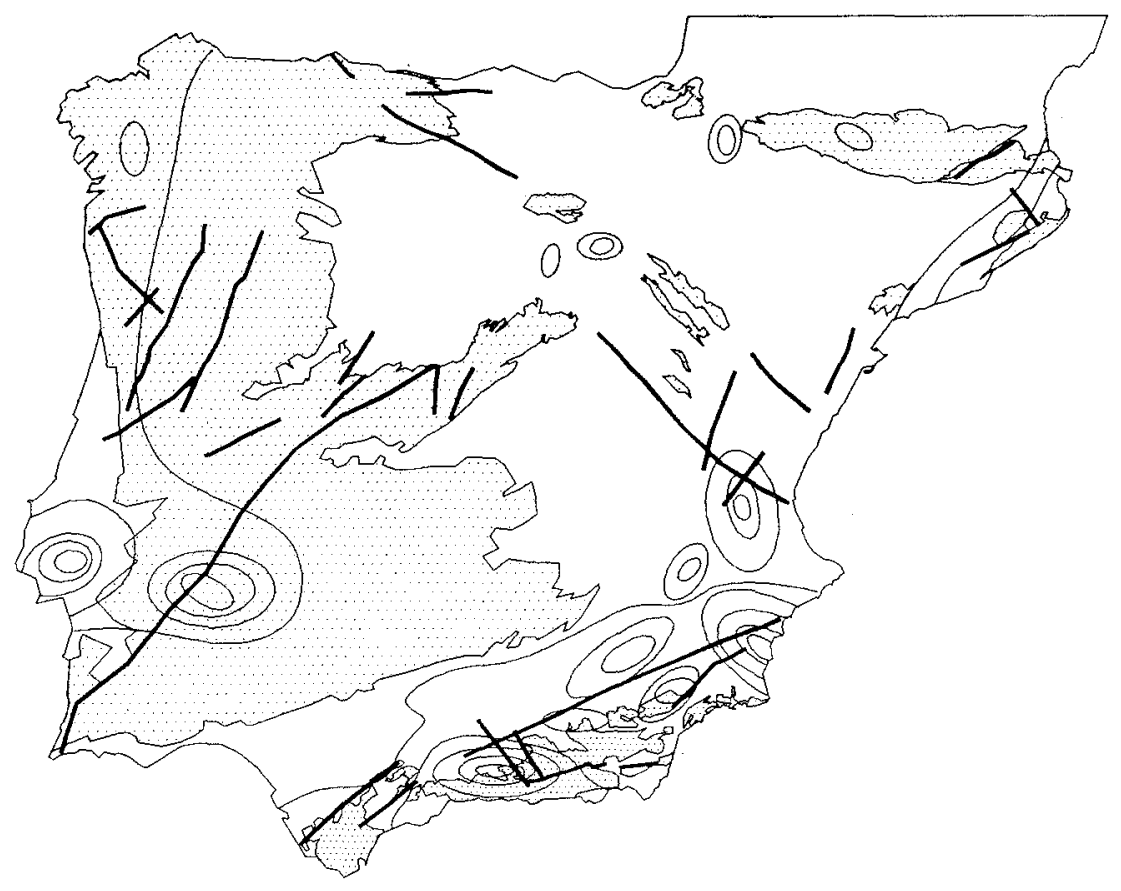

Fig. 1. Mapa-base utilizado en las figuras de este trabajo. En él se ha llegado al compromiso, ciertamente dificil, de representar los rasgos geológicos indispensables para hacer posible la visualización de la posible relación entre algunos fenómenos tectónicos y la distribución de los A.M.M.T. que se consideren en cada caso. Se ha señalado el conjunto del zócalo hercínico (granitico y metamórfico) que ocupa prácticamente la mitad occidental de la península y áreas pirenaicas (relleno de puntos); diferenciándolo en una trama sólo un poco desigual de las rocas del mismo tipo existentes en la zona axial de la Cordillera Bética.

En blanco se han dejado las áreas ocupadas por los depósitos mesozoicos y cenozoicos, a'gunos de ellos plegados por la Orogenia Alpina. Con trazo grueso se han marcado algunas fallas o fracturas seleccionadas y que se considera deben estar incluidas en cualquier intento de sintesis de este tipo, lógicamente no se han representado todas las fallas o fracturas existentes en la peninsula.

También se han señalado con trazo fino y continuo las isosistas que recogen las zonas de intensidad decreciente desde $X$ hasta VI según los datos del Mapa Sismoestructural (I.G.M.E.-I.G.C. 1966) de la península Ibérica. 
la relación y señalización de estos fenómenos en un apartado desitinado a Hidrología e Hidrogeología. También, lógicamente, las monografías realizadas al respecto y diversos artículos publicados en revistas científicas. Algunos trabajos de recopilación merecen reseñarse especialmente, como los efectuados por LOPEZ DE AZCONA (1956); LOPEZ DE AZCONA et al. (1948, 1953); Cruz-Sanjulián et al. (1975); Albert I Beltran et al. (1985) e I.G.M.E. (1986). Todos ellos se citan en las Referencias, aunque por su volumen las mencionadas ediciones del I.G.M.E. a escala 1:50.000 aquí sólo se recogen globalmente. Para Portugal se han consultado igualmente las que creemos son todas las Cartas Geológicas a Escala 1:50.000 publicadas hasta la fecha por el Servicio Geológico de Portugal, en los que son frecuentes las referencias a estos temas. Como en el caso de España su referencia se hace aquí de forma genérica. Para Francia se ha hecho uso de la recopilación de LANCE (1987). Partiendo de dicha Base de Datos se han realizado los mapas de distribución que ahora sirven de apoyo a este análisis.

\section{DISTRIBUCIÓN DE LAS FUENTES MINERO-MEDICINALES}

La situación de todos los 1.592 puntos para los que se ha encontrado localizado una referencia de A.M.M.T. (fig. 2) manifiesta una distribución no homogénea, selectiva. Dentro del conjunto Hercínico la región NW en el borde atlántico y en la zona costero-catalana, son las áreas donde se manifiestan acumulaciones importantes. Cabe señalar, asimismo, los acumulados existentes en la región volcánica de los Campos de Calatrava (Ciudad Real).

En la superficie ocupada por los depósitos meso-cenozoicos las acumulaciones de puntos más importantes se dan en el País Vasco, en la zona costero-catalana y en la Bética; también son de destacar las existentes al sur de Madrid y en los Pirineos.

Observamos cómo, en general, las pautas de alineaciones o acumulaciones de puntos con A.M.M.T. tienen que ver con estructuras o caracteres geológicos de orden decakilométrico. Así vemos cómo las acumulaciones de puntos en Galicia y $\mathrm{N}$ de Portugal se manifiestan en relación a alineaciones NE-SW de longitud del orden de 60-70 km. También en esos órdenes de longitud se alinean los puntos del País Vasco, Cantabria y Béticas, etc. Por el contrario, hay estructuras geológicas conocidas de orden de magnitud hectokilométrica, como, por ejemplo, Ia Falla de Pla- 


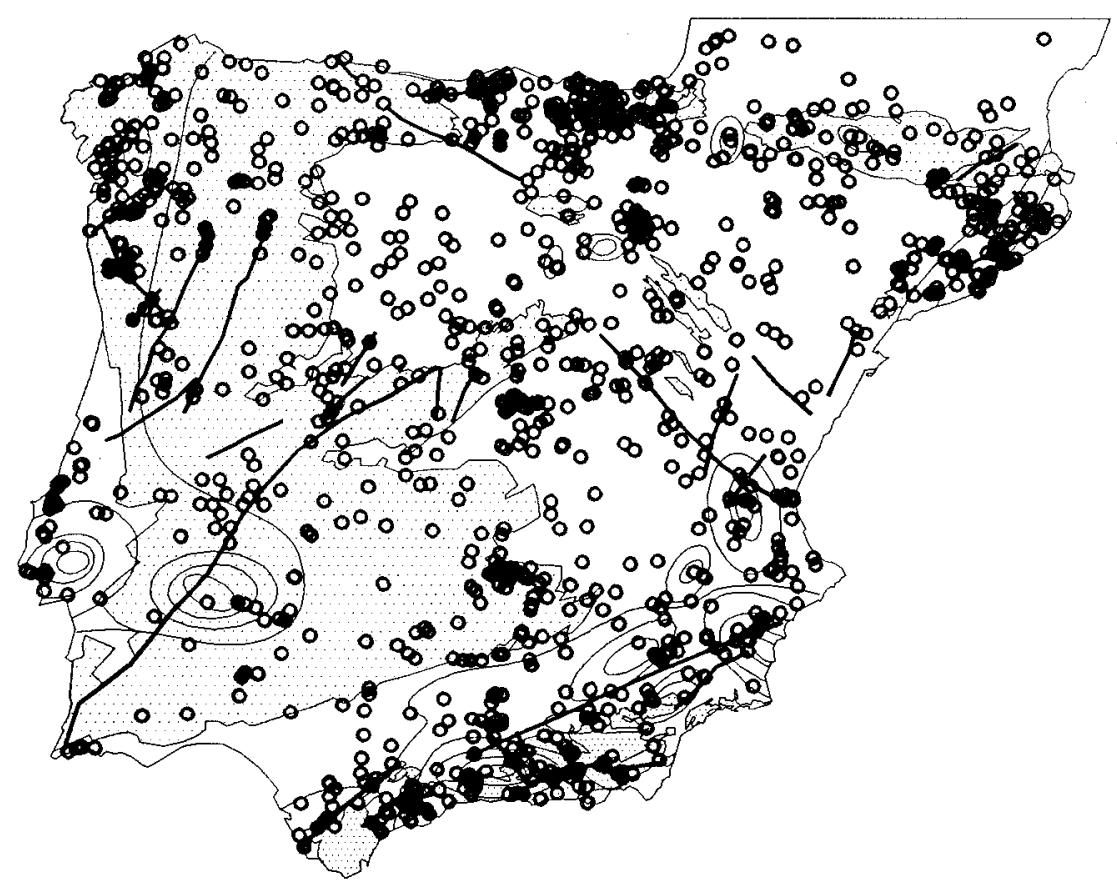

Fig. 2. Distribución sobre el mapa-base del total de 1.592 puntos fuentes mineromedicinales y/o termales localizados hasta la fecha en la península lbérica y sur de Francia.

sencia (desde Cabo de San Antonio a Ávila), en relación a la cual se encuentran algunos puntos, pero formando acumulados de amplitud menor.

En cuanto a la relación entre los puntos A.M.M.T. con las áreas sísmicas las de la figura 2 señala cómo son ciertas estas ligazones en Cataluña y Béticas, sobre todo en Granada-Málaga, también al norte de Lisboa y Badajoz. En otros puntos hay coincidencias, como por ejemplo en La Rioja, sur de Sierra de la Demanda y Pirineos centrales, pero no hay acumulados.

DISTRIBUCIONES SELECTIVAS POR TEMPERATURAS

Parece de interés que al tratar del fenómeno de A.M.M.T. podamos discriminar algunas localizaciones en función precisamente de la tempe- 
ratura que tienen las aguas en su punto de surgencia. Para ello se han elegido los límites de las temperaturas que se utilizan en el I.T.G.E. para la clasificación de las aguas según este factor (I.G.M.E. 1986). Son ya conocidos algunos de los problemas reales (RAmos OntIVERos 1975; CruzSANJULIÁN 1976), que la utilización de unos límites estrictos conlleva cuando se trata de aplicar esta división a todas las zonas de una región amplia como la que aquí estamos tratando. Sin embargo, para nuestros fines los límites utilizados pueden ser tan revisables como cualesquiera otros que se hubieran seleccionado y coinciden con los aceptados por la generalidad.

Mapas de puntos «fríos»

En hidrología se consideran aguas «frías» las que surgen a menos de $20^{\circ} \mathrm{C}$. En la península tenemos localizados 1.194 de estos puntos (fig. 3).

Las surgencias de temperaturas «frías» tiene una distribución que se

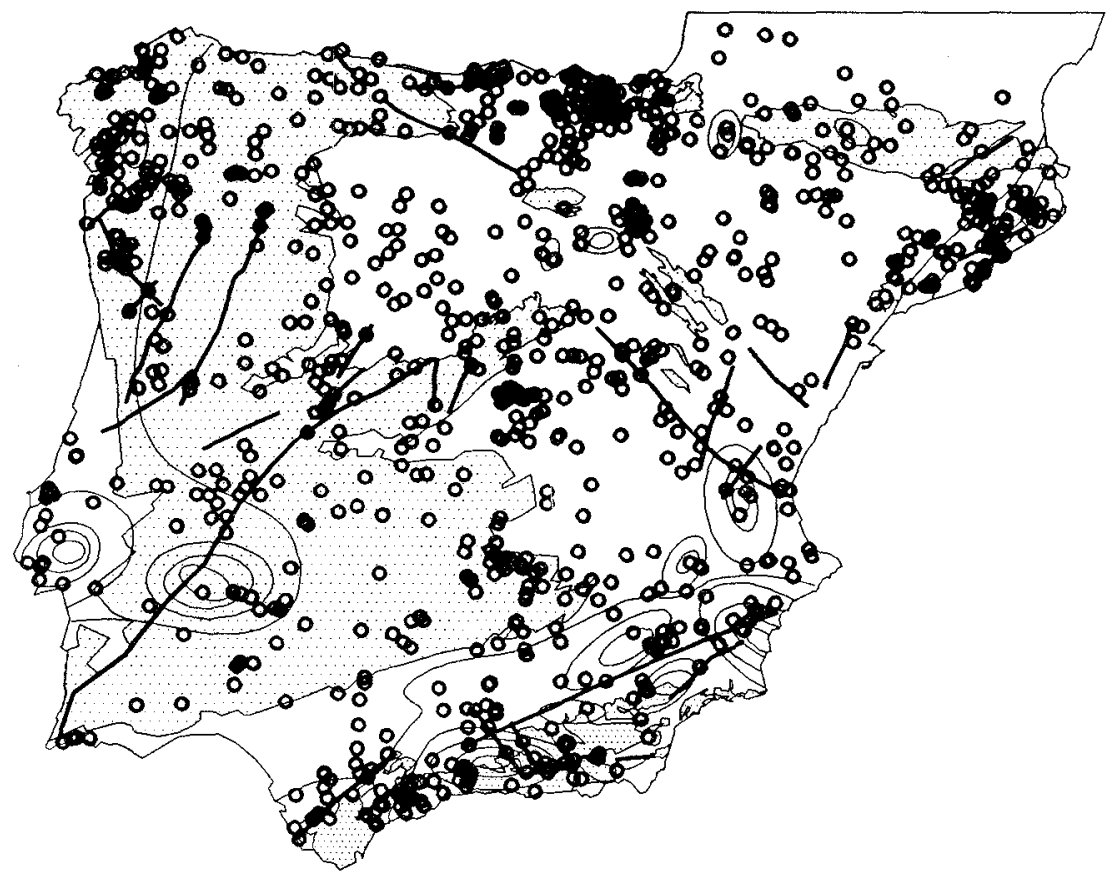

Fig. 3. Distribución de los 1.194 puntos termales «frios», es decir, con temperatura de surgencia menor a los $20^{\circ} \mathrm{C}$. 
asemeja a la encontrada en la figura 2 referente al conjunto de todos los puntos A.M.M.T. localizados en la peninsula Ibérica. Esta casi superposición no es de extrañar dado el número próximo de puntos existentes en ambos conjuntos (1591 y 1194, respectivamente) y la frecuente circunstancia de que en la misma localidad donde se halla una surgencia termal suele haber, asimismo, otra $u$ otras con temperatura más baja. Como se aprecia en la figura 2, estas localizaciones muestran una doble preferencia: la de situarse alineadas sobre fallas transversales a las cadenas montañosas (Béticas, Pirineos y Costero-Catalana); formar alineaciones decakilométricas NE-SW, como en Galicia y País Vasco, y NW-SE en Cataluña, que enlazan con las del Campo Volcánico de Olot (RIBA 1975) según un enjambre de fracturas (FERNÁNDEZ Y BANDA 1989), y desarrollarse bien sobre los grandes accidentes peninsulares como la del límite meridional del zócalo de la Meseta (casi coincidente con el río Guadalquivir), falla de Alicante-Cádiz y borde de la cordillera Costero-Catalana.

Mapa de puntos «hipotermales»

Es decir, los puntos en los que las aguas surgen a temperaturas entre los 20 y $30{ }^{\circ} \mathrm{C}$. Con esta característica tenemos localizados 213 puntos A.M.M.T. en la península lbérica (fig. 4). La mayoria se encuentran en la mitad sur del borde mediterráneo español, en estrecha relación tanto con fallas como con áreas de sismicidad importante.

No es de extrañar que también se distribuyan con cierta frecuencia en los alrededores del área volcánica de los Campos de Calatrava y áreas sísmicas y volcánicas del Tajo en Portugal.

Los puntos de Jaén y Córdoba (como ya señalaron CRUZ-SANJULIÁN y Garcia-Rossell 1975) están alineados sobre el accidente tectónico que separa el macizo Hercínico del Prebético (Falla del Guadalquivir) y también se marcan las líneas $N 140^{\circ}-150^{\circ}$ en los puntos de Granada y Málaga que constituyen fallas transversas a la cordillera Bética (Op. cit.). En el Pirineo se alinean a grosso modo con el eje longitudinal de la cordillera, adaptándose a contactos y fallas que ponen en relación los zócalos hercínicos con la cobertera meso-cenozoica y también se alinean sobre el accidente que con dirección SW-NE transecta los pirineos centrales. Al sur de Cantabria y País Vasco se alinean en la dirección NW-SE, la misma que las fallas allí señaladas. 


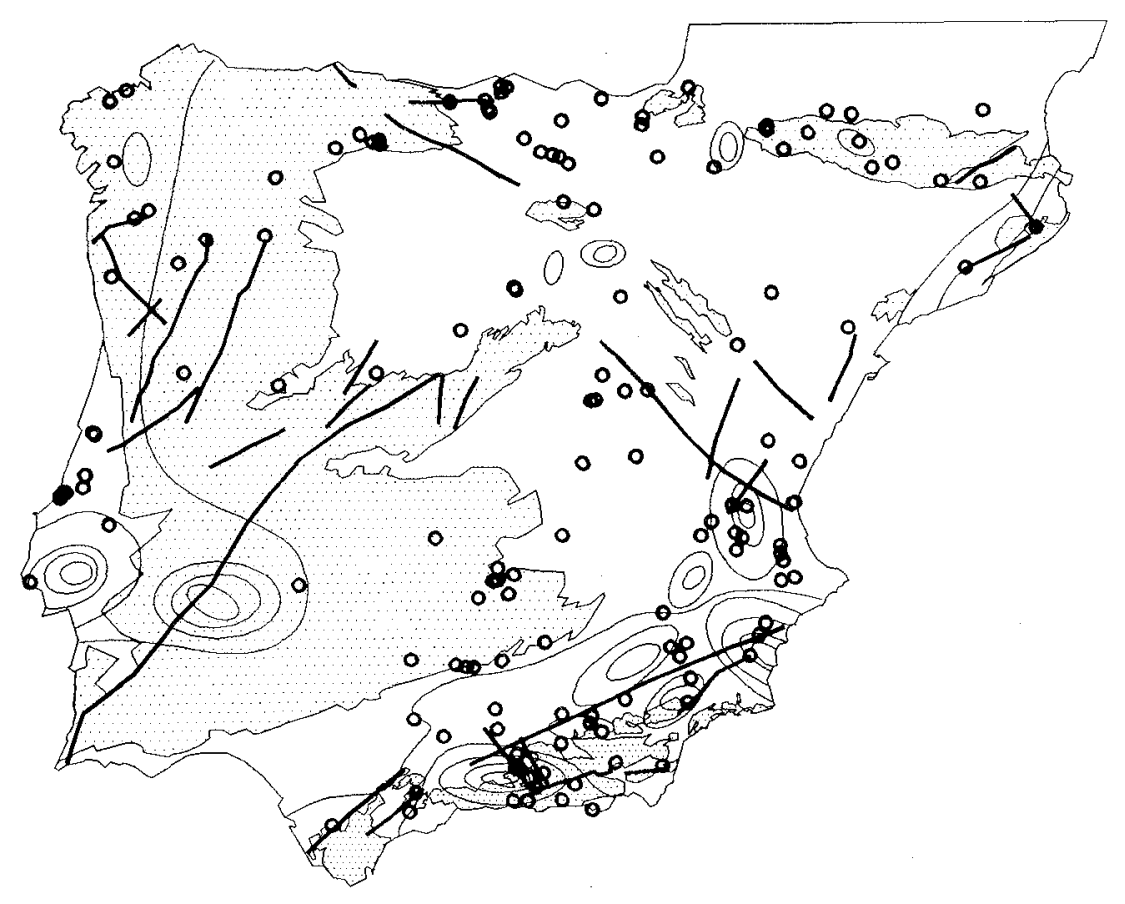

Fig. 4. Distribución de ios 213 puntos hipotermales, es decir, con temperaturas de surgencia entre los 20 y $30^{\circ} \mathrm{C}$.

Mapa de puntos «mesotermales»

Son las surgencias con temperaturas entre 30 y $40{ }^{\circ} \mathrm{C}$. Lógicamente hay menos puntos que en los casos anteriores. Se han localizado 86 y presentan una distribución muy selectiva (fig. 5). Áreas ya citadas en las Béticas, Cataluña, Pirineos y desembocadura del Tajo, son los lugares destacados. Y aquí además hay que señalar la zona de Galicia que aumenta su número con relación al de puntos hipotermales.

Para las áreas mediterráneas y atlánticas es evidente la vinculación entre la localización de muchos de estos puntos y la de focos sísmicos de máxima intensidad. Para el interior de la Meseta, con muy pocos puntos, cabe señalar su exacta localización sobre fallas importantes y/o contactos por falla entre los correspondientes zócalos hercínicos y la cobertera meso-cenozoica. 


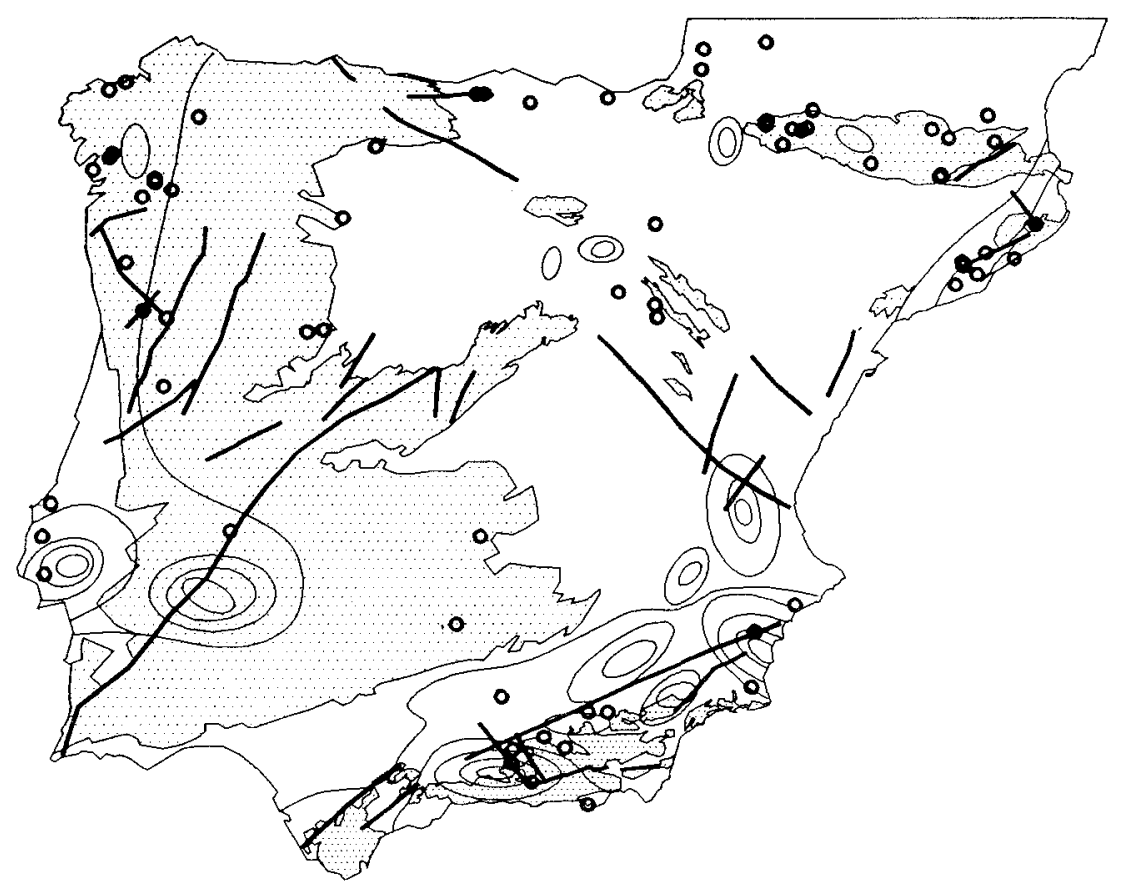

Fig. 5. Distribución de los 86 puntos mesotermales, con temperaturas en la surgencia entre los 30 y $40{ }^{\circ} \mathrm{C}$.

Mapa de puntos «hipertermales»

Con temperaturas a más de $40^{\circ} \mathrm{C}$. Tenemos localizados 98 puntos con estas características (fig. 6). Presentan una distribución más selectiva aún que las anteriores y reflejan lo que podemos considerar son las áreas de mayor potencial geotérmico de la zona peninsular: los Pirineos y áreas costero-catalanas; Béticas y áreas alicantino-murcianas; Galicia y Salamanca, son las principales áreas. Cerrando el conjunto están los puntos aislados de La Rioja, Asturias y sur de Francia, tanto en su vertiente mediterránea como atlántica.

Se hallan en relación con áreas sísmicas de fuerte intensidad, con fallas y contactos entre el zócalo y cobertera sobre todo en los bordes de zonas axiales de cordilleras.

En relación con estos puntos de máxima temperatura se puede comentar la sorprendente ausencia de los mismos en la zona del País Vasco donde se han detectado importantes acumulaciones de surgencias. Un punto de estas características (La Garriga, en el Vallès-Penedés) estudia- 


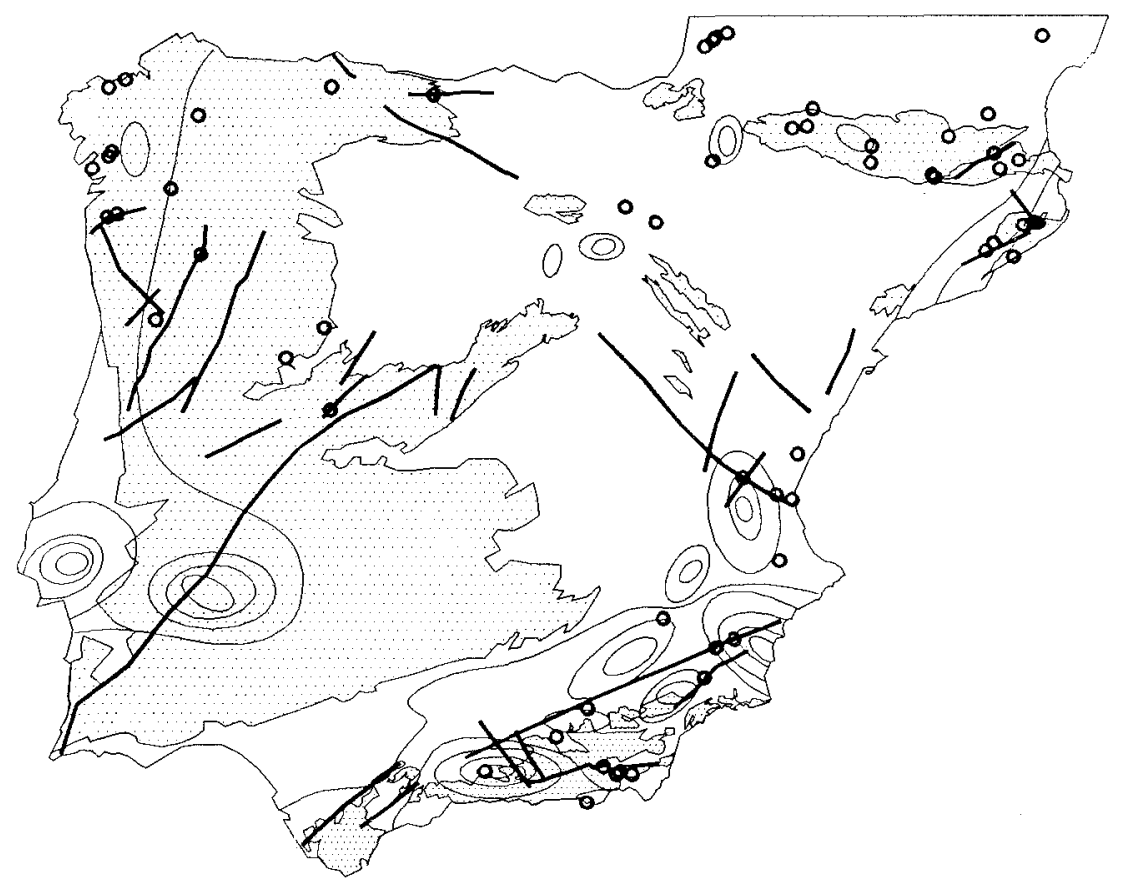

Fig. 6. Distribución de los 98 puntos hipertermales, con temperaturas superiores a $40{ }^{\circ} \mathrm{C}$.

do con más detalle muestra que la causa de la existencia de este fenómeno es debida a un proceso convectivo en la circulación del agua meteórica que es forzada a través de una doble circunstancia allí existente: una falla profunda (borde norte del Graben del Vallès-Penedés) y un contacto entre granito y rocas metamórficas, ambos hercínicos; la conjunción de todos estos factores provoca esta manifestación hipertermal puntual en un área con gradiente geotérmico general «normal» (FERNANDEZ Y BANDA 1990).

\section{DISTRIBUCIÓN POR ALGUNOS CARACTERES QUÍMICOS}

Sin pretender ser exhaustivos y simplificando las determinaciones que pueden llegar a ser más concretas (ver, por ejemplo: I.G.M.E. 1986), se exponen aquí la distribución de los puntos de surgencias de A.M.M.T. 
atendiendo a algún carácter químico que hemos recogido en la Base de Datos. Estas características las hemos incluido de forma cualitativa no cuantitativa, por lo que los resultados que se ofrecen deben ser tomados con esta salvedad.

\section{Aguas radioactivas}

El número de surgencias radioactivas que se señalan en los datos existentes hasta ahora es de 181. Por el tipo de parámetros a medir para saber si un agua es radioactiva o no se puede suponer que este número será mayor, aunque no es posible decir en qué medida.

Su distribución (fig. 7) indica que se encuentran principalmente en Galicia, sur del País Vasco-norte de Burgos, Pirineo y Cataluña; en Portugal en las proximidades de Lisboa. El conjunto más concentrado y numeroso se encuentra en Cataluña. Sin embargo, la surgencia conocida más ra-

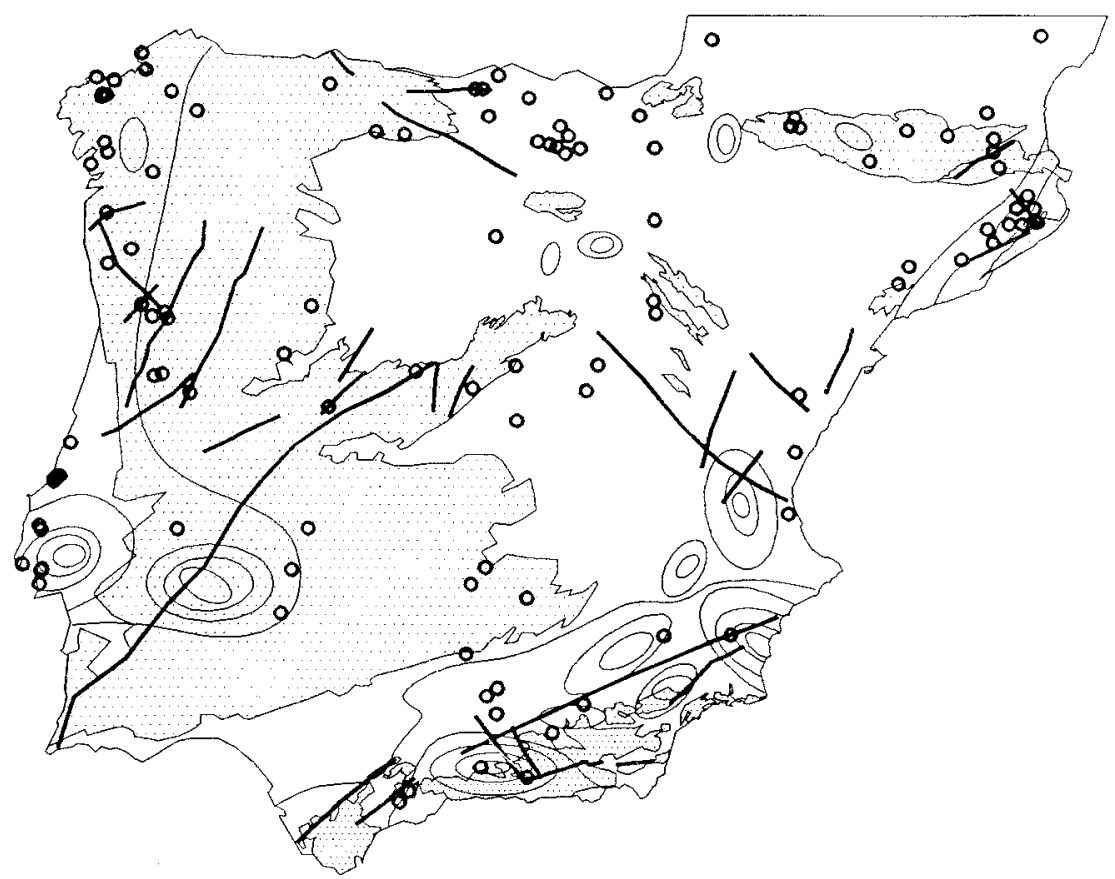

Fig. 7. Distribución de los 181 puntos en que se tienen noticias de tener un carácter radioactivo. 
dioactiva de la península se encuentra en las cercanias de Valdemorillo (Madrid) en el conjunto granítico del Sistema Central.

Aguas ferruginosas

Con este carácter se han localizado 447 puntos (fig. 8). Concentradas principalmente en el País Vasco y región cantábrica, también son de destacar las alineaciones entre Málaga y Granada, Galicia, región volcánica de Campos de Calatrava y alineaciones longitudinales según la cadena Costero-Catalana.

Otros puntos en el macizo Hercínico (Extremadura y Zamora) están relacionados en un caso con la Falla de Plasencia y en otro con el contacto entre el zócalo y la cobertera.

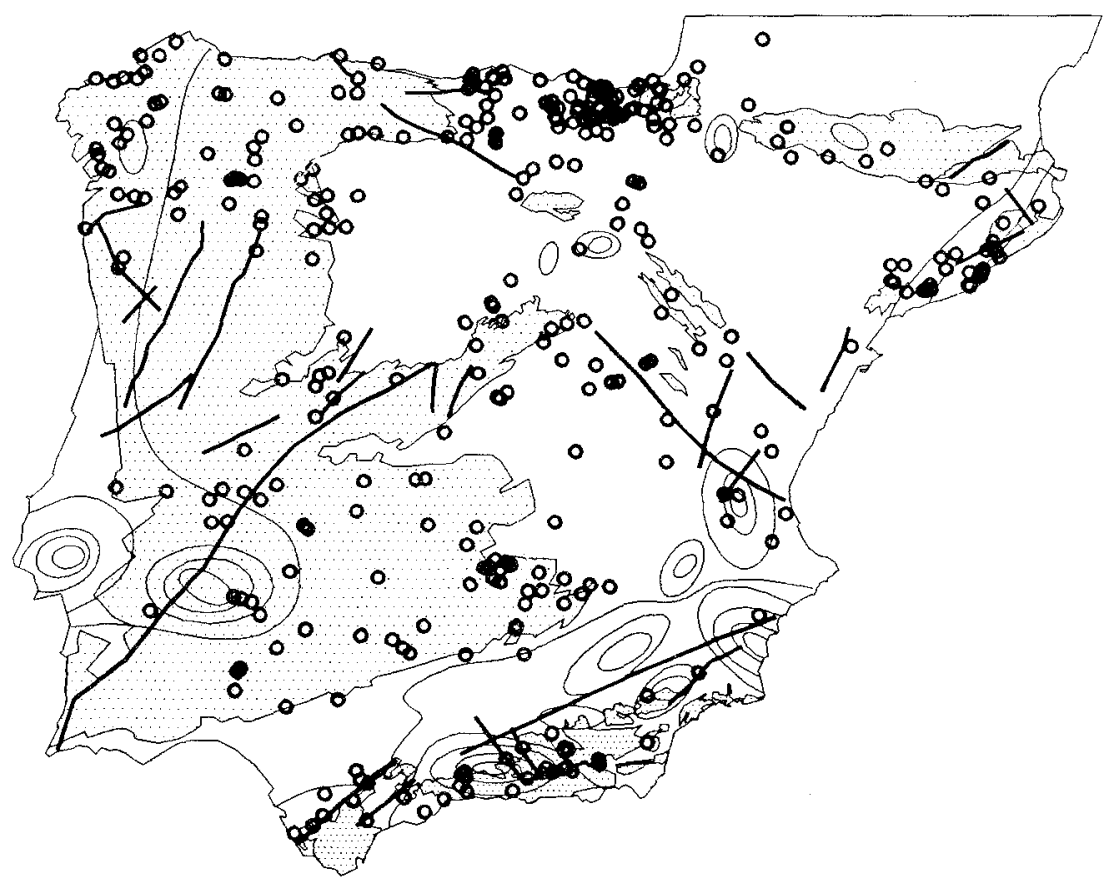

Fig. 8. Distribución de los 447 puntos de fuentes «ferruginosas» con caracteres químicos anómalos en hierro. 
Aguas «bicarbonatadas»

Se indican un total de 491 surgencias (fig. 9). Se presentan acumuladas en áreas restringidas, como por ejemplo, casi únicamente en la zona sur de Galicia, alineadas SW-NE en el Pais Vasco y NW-SE en las Béticas, Campos de Calatrava, según N-S en Valencia-Alicante y longitudinalmente a la cadena costera en Cataluña.

\section{Aguas «sulfurosas»}

Según estos caracteres químicos son las más numerosas, con un total de 691 registros (fig. 10).

Ya con este número se puede esperar una distribución próxima a la del conjunto total de surgencias de la figura 2. $Y$ en efecto las dos distribuciones guardan gran semejanza, por lo que cabe en este caso destacar

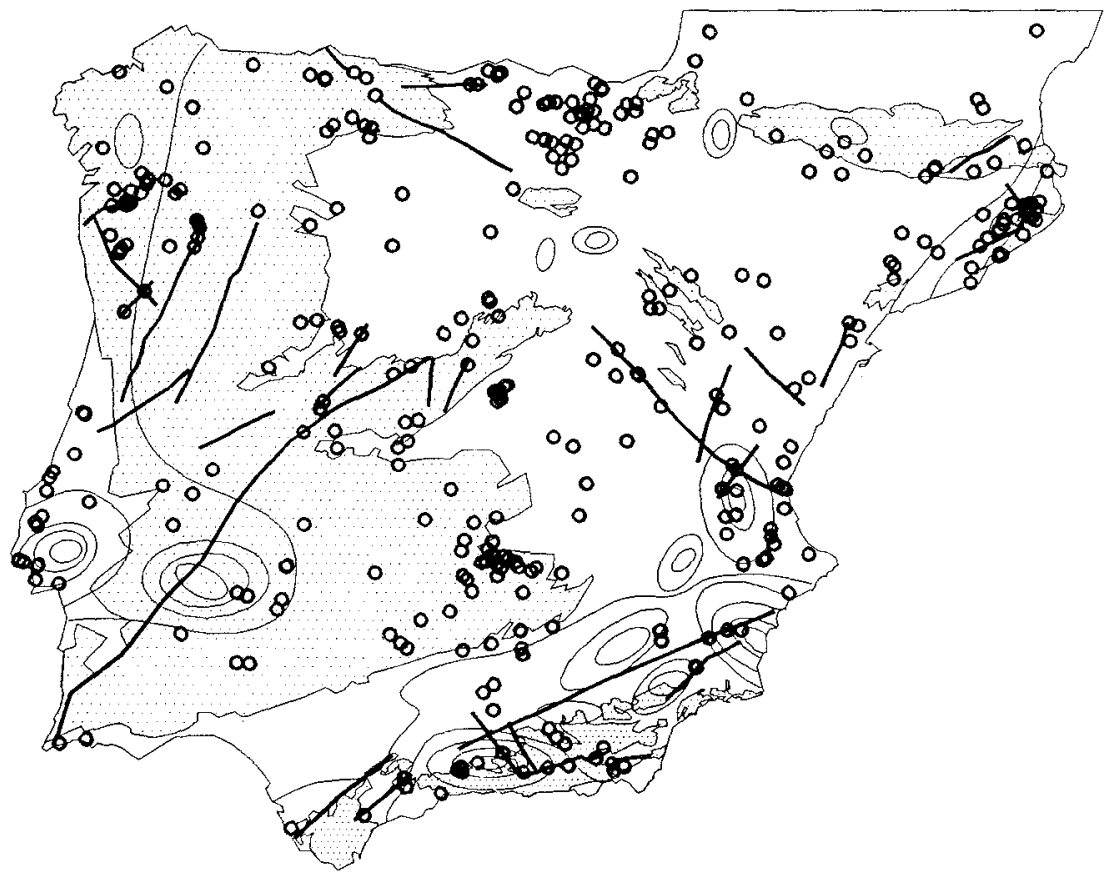

Fig. 9. Distribución de los 491 puntos surgentes de aguas con caracteres anómalos en su contenido de elementos que de forma simplificada hemos englobado en «bicarbonatados». 


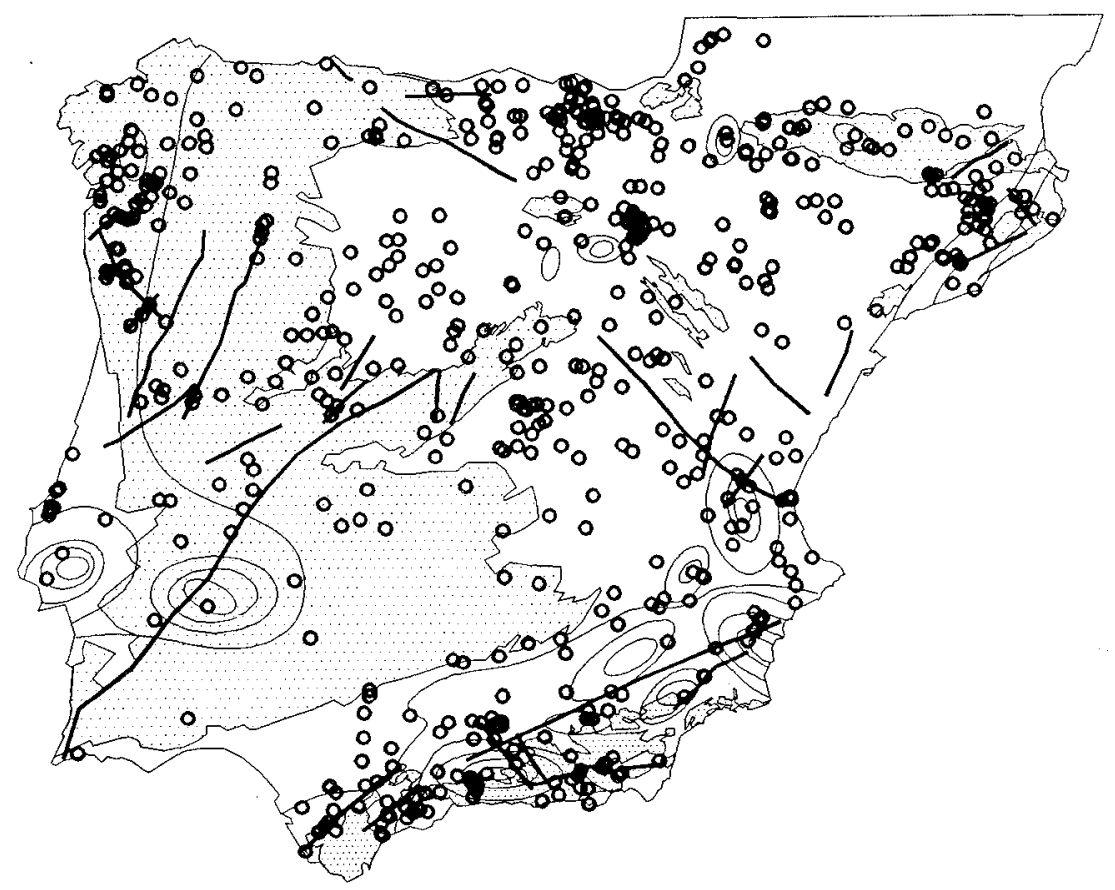

Fig. 10. Distribución de los 691 puntos de surgencia de aguas con caracteres anómalos en su contenido de elementos que de forma simplificada hemos englobado como «sulfurosos».

las diferencias, que se refieren a las ausencias de puntos con este carácter en la región volcánica de los Campos de Calatrava (Ciudad Real), borde costero de Cataluña y también se puede mencionar el norte de Galicia.

RELACIÓN ENTRE LA DISTRIBUCIÓN DE LAS AGUAS TERMALES Y LA ESTRUCTURA GEOLÓGICA DE LA PENINSULA IBÉRICA

Para la generalidad de la península lbérica la relación entre el flujo geotérmico y la estructura y tectónica han sido analizados y se muestra como manifiesta (Albert y BANDA 1984). Por otra parte, se sabe que otro factor a tener en cuenta (aunque todos ellos, asimismo, están a su vez interrelacionados) es la correlación inversa que tiene el valor de este flujo con el del espesor de la corteza, de tal manera que los máximos flujos 
caloríficos tienen lugar en las áreas de la corteza que tienen mínimo espesor (Albert-Beltrán 1979).

Los puntos que se encuentran en las cercanías del borde Mediterráneo español forman una cadena de fenómenos que pueden enlazarse con los que se observan en Francia y Alemania (LUCAZEU Y VASSER 1989), definiendo todo ello una estructura en la dirección norte-sur que se prolonga desde la Fosa del Rhin y Macizo Central francés hasta Cabo de Gata (SE de España). Esta gran estructura europea corresponde a un rift abortado que se desarrolló incipientemente durante el Cenozoico (JULIVERT et al. 1974). Muchos puntos de esta franja mediterránea muestran correspondencia y evidente superposición con áreas actualmente sísmicas (no sucede otro tanto con las áreas volcánicas de Olot y Cabo de Gata). En detalle puede verse que las surgencias calientes están a su vez en relación con la presencia de fallas o fracturas de orden longitudinal de decakilómetros, la mayoría de las veces transversales a la dirección estructural de la respectiva cadena orogénica.

Conviene seguir la búsqueda de nuevos datos sobre la presencia de puntos con estas características, pues aunque la muestra aquí analizada es amplia, debemos suponer que sólo es una parte, no sabemos en qué medida, del total que podamos manejar en el futuro.

\section{RESUMEN}

En base a la consulta de un amplio material bibliográfico se ha podido elaborar un amplio fichero informático referente a las surgencias de aguas minero-medicinales y/o termales (A.M.M.T.) de la península Ibérica.

Por medio del uso de sistemas informáticos se han realizado mapas geológicos-geográficos que nos muestran la distribución de estas surgencias en función de algunos caracteres químicos y de su temperatura.

La distribución de los puntos A.M.M.T. en la península lbérica no es homogénea; podemos considerar que la concentración en áreas o regiones es uno de sus rasgos característicos. Algunas de estas acumulaciones (por ejemplo, en la región de volcanismo cuaternario de Campos de Calatrava) se da en función del carácter químico que se considere.

Las aguas termales parecen tener preferencia por las regiones del borde mediterráneo donde se encuentran una gran parte de surgencias con temperaturas mayores a $20^{\circ} \mathrm{C}$. En el País Vasco se da la concentra- 
ción más numerosa de surgencias A.M.M.T., aunque alli son escasos los valores de temperatura más altos de $20^{\circ} \mathrm{C}$.

Para el conjunto peninsular se observa una buena coincidencia entre la distribución regional de las A.M.M.T. y la existencia de fracturas o fallas de longitud del orden de $60 \mathrm{~km}$. Esta escala es la que parece actuar de control en la distribución de estas surgencias. En las fracturas o fallas de mucha mayor magnitud no se presentan fenómenos de este tipo en toda su extensión, quedan localizados en regiones puntuales o regiones con acumulaciones del orden ya mencionado.

También en las regiones de la orla mediterránea es donde se observa la mayor correlación en la superposición de puntos A.M.M.T. y actividad sísmica.

\section{ABSTRACT}

In base to the consultation of an ample published datasets it has been able to process a referring file to the springs of thermal and/or mineral waters (A.M.M.T.) of the Iberian Peninsula.

By means of the use of computer systems it has realized geologicgeographic maps that show us the distribution of these spring waters in function of some chemical caracters and of its temperature.

The distribution from the 1592 points A.M.M.T. in the Iberian Peninsula is not homogenous; it's possible to consider that the concentration in areas or regions is one of their characteristics. Some of these accumulations (for example in the volcanic region quaternary of Fields of Calatrava) it gives in function of the chemical character that it considers.

The thermal waters resemble to have preference for the regions of the edge Mediterranean where a big part of springs with major temperatures to $20{ }^{\circ} \mathrm{C}$ are found. In the Vasco Country it gives the concentration more numerous of A.M.M.T., although there are scarce values of temperature higher of $20^{\circ} \mathrm{C}$.

To the peninsular join it is observed a good coincidence between the regional distribution from the A.M.M.T. and the existence of fractures or faults of longitud of the order of $60 \mathrm{~km}$. This scale is the one that seems to act of control in the distribution of these springs. In the fractures or faults of much magnitude are not present phenomenons of this type in all its extension, they remain located to punctual regions or regions with accumulations of the now mentioned order. 
Also in the regions of the mediterranean littoral is where it is observed the major correlation in the overposition of points A.M.M.T. and sismic activity.

\section{REFERENCIAS}

Albert Beltrán, J. F. (1979): «El mapa español de flujos caloríficos. Intento de correlación entre anomalías geotérmicas y estructura cortical», Bol. Geol. Min., 90, págs. 36-48. Madrid.

Albert, J. F., y Banda, E. (1984): «España en el contexto geotérmico europeo. Áreas de interés», en Energía Geotérmica. Madrid, Inst. Ingeniería de España. Edit. I.G.M.E., págs. $37-47$.

Albert I Beltrán, J. F.; Aragonés, E.; Bayo, A.; Corominas I Dulcet, J.; Corominas I Blanch, J.; Mata-Perello, J. M., y Riba I Arderiu, O. (1985): "Les manifestaciones termales", en Recursos Geològics i Sòl. Historia Natural dels Països Catalans. Barcelona, Edit. Enciclopedia Catalana, S. A., págs. 204-213.

Enciclopedia Universal llustrada. Tomo III (1930): «Aguas minerales». Barcelona, Edit. J. Espasa e Hijos. Barcelona, págs. 562-597.

Guia oficial de los establecimientos balnearios y aguas medicinales de España. Año 1927. Madrid, Edit. Rudolf Mosse, 360 págs.

Benavente, J., y SANZ DE GaldeAno, C. (1985): «Relación de las direcciones de karstificación y del termalismo con la fracturación en las Cordilleras Béticas», Estudios Geológicos, 41, págs. 177-188. Madrid.

Cruz-Sanjulián, J., y Garcia-RosselL, L. (1975): “Termalismo en España meridional», Bol. Geol. Min., 86, págs. 179-186. Madrid.

Cruz-Sanjulián, J.; Garcia-Rossell, L., y Garrido-Blasco, J. (1972): "Aguas termales de la provincia de Granada", Bol. Geol. Min., 83, págs. 266-275. Madrid.

Cruz-Sanjulian, J. (1976): "Sobre la temperatura límite del termalismo", Tecniterrae, 12, págs. 45-54. Madrid.

FERNÁNDEZ, M., y BANDA, E. (1989): «An approach to the thermal field in northeastern Spain», Tectonophysics, 164, págs. 259-266. Amsterdam.

- (1990): "Geothermal anomalies in the Valles-Penedes graben master fault: convection through the horst as a possible mechanism», Journal Geophysical Research, B4, 95, págs. 4887-4894. Washington.

HeRnÁNDEZ-PACHECO, F. (1949): La tectónica peninsular y su relación con las aguas mineromedicinales. Madrid, Real Academia de Farmacia. Disc. Recepc. 134 págs.

I.G.M.E.-I.G.C. (1966): Mapa sismoestructural de la peninsula Ibérica, Baleares y Canarias. Escala 1:1.000.000. Madrid.

I.G.M.E. (1986): Las aguas minero-medicinales, minero-industriales y de bebida envasadas existentes en España. Estudio preliminar. Madrid, Informe. 140 págs.

Julivert, M.; FontBoté, J. M.; Ribeiro, D., y ConDE, L. (1974): Mapa Tectónico de la península Ibérica y Baleares. Escala 1:1.000.000. Memoria explicativa. 133 págs. Madrid, Edit. I.G.M.E.

Lance, PH. (1987): Le Thermalisme practique. Guide. La Chapelle Montligeon. Francia.

López de Azcona, J. M.; Martin Cardoso, G., y Parga Pondal, I. (1953): «Explicación de la Hoja N 261. Tuy (Pontevedra)", Mapa Geológico de España. Escala 1:5.000. Madrid, I.G.M.E. 94 págs.

lopez de Azcona, J. M.; Hernandez Sampelayo, A., y lizaur y Roldán, J. de (1948): «Explicación 
de la Hoja N 21. La Coruña», Mapa Geológico de España. Escala 1:5.000. Madrid, I.G.M.E. Madrid. 101 págs.

- (1956): "Las aguas minero-medicinales de la provincia de Pontevedra", Notas y Comunicaciones I.G.M.E., 41, págs. 5-20. Madrid.

- (1963): «Las aguas minero-medicinales. Su industrialización», Bol. Inst. Geol. Minero de España, 74, págs. 337-392. Madrid.

Lucazeau, F., and Vasser, G. (1989): "Heat Flow density data form France and surrounding margins», Tectonophysics, 164, págs. 251-258. Amsterdam.

Menduiña Fernández, J. (1978): “Geotermismo y mineria», Bol. Geol. Min., 88, págs. 564-570. Madrid.

Mezcua, J., y Martinez Solares, J. M. (1983): Sismicidad del área ibero-mogrebi. Pb. 203. Madrid, Inst. Geograf. Nacional. 299 págs.

Ramos Ontiveros, I. M. (1975): «Criterios de clasificación de aguas termales», Tecniterrae, 7 , págs. 34-36. Madrid.

RIVA, O. (1975): "Geotermismo de la zona volcánica de Olot. Nota preliminar sobre posibilidades geotérmicas", Bol. Geol. Min., 86, págs. 45-62. Madrid.

SÁnchez LozANO, R. (1914): "La tectónica general en sus relaciones con las agua mineromedicinales», Bol. Inst. Geol. España, 34, 1914 (1913), págs. 295-305. Madrid. 